\title{
Characterization of Reconstructed Basins Using Pattern Spectrum Procedure
}

\author{
P. Radhakrishnan \\ Faculty of Information Science and Technology \\ Multimedia University, Bukit Beruang, Melaka, 75450, Malaysia
}

\begin{abstract}
Several classical and Fractal binary shapes, which are akin to geophysical shapes such as basins, lakes, and pore-grain spruce, are analyzed and characterized by employing various mathematical morphological transformations, and methods. By employing rhombus, square and octagon structuring elements, these shapes are decomposed into their skeletal networks and their corresponding skeletal network subsets are dilated to the respective degree by these structuring elements in order to reconstruct the original shapes. Furthermore, to test the reconstruction accuracy, the pattern spectrum procedure is applied and sharpness indices were computed. These shapiness indices were considered as a basis to test the reconstruction accuracy in a quantitative manner. A general trend is observed while characterizing the shape-size complexity of these surface water bodies.
\end{abstract}

Key words: Geophysical shapes, reconstruction, mathematical morphology, pattern spectrum, shapesize complexity

\section{INTRODUCTION}

Shape description is an important issue in understanding various geophysical processes. The examples of shapes that are encountered in geophysical sciences include basins, elevation regions, lakes, rock pore-grain space etc. Investigation of such shapes has significant scope from the point of geophysical studies. There are certain indicators that determine the overall shape from the geophysical context. These indicators are the unique patterns such as the channel network, flow direction network, and the pore-grain connectivity networks that determine the shapes of basins, lakes, and pore-grains respectively. These unique network patterns can be treated as minimum morphological information employing which one can make an attempt to reconstruct the original shapes. For instance, the spatial organization of the channel network patterns determines the basin processes. This channel network pattern, we call as a minimum, but highly significant, morphological information, from which one can reconstruct the basin. Employing various morphological rules and fundamental morphological transformations can do this. To verify the accuracy of the reconstructed basin by comparing with original basin organizations in spatial domain, the pattern spectrum procedure is of immense use. However, in this paper, we have considered various synthetic images in binary form, which are akin to threshold elevation regions of a typical Digital Elevation Model (DEM) ${ }^{[1]}$. These synthetic shapes were converted into possible connectivity network patterns, which are akin to the minimum morphological information in the basin, i. e., Channel network patterns. These connectivity network subsets are our main focus from which we tried to reconstruct the original shapes. To test the accuracy in the reconstructed region, by employing various predefined morphological rules, we have adapted pattern spectrum procedures. This study will have significance with greater insight in evaluating the reconstruction of various geophysical phenomena such as basins, lakes, pore-grain space etc.

Morphological Transformations: Mathematical morphology ${ }^{[2,3]}$ is a set algebra used to process and analyze data based on geometric shapes. It examines the geometrical structure of an image by probing it with small patterns, called structuring element (SE). The discrete binary image, $\mathrm{X}$ is defined as a finite subset of Euclidean two-dimensional space $Z^{2}$. Let $B$ denote a structuring element, which is a subset in $Z^{2}$ with a simple geometrical shape and certain characteristic information. The morphological operators can be visualized as working with two images. The image being processed is referred to as the image and other image being a structuring element. The four basic morphological transformations are eroded, dilation, cascade of erosion-dilation and dilation-erosion. The morphological erosion of a basin, $\mathrm{X}$ with structuring element, $\mathrm{B}$, is defined as the set of points $\mathrm{m}$ such that the translated $B_{m}$ is contained in $X . \quad X \quad \ominus$ $\mathrm{B}=\left\{\mathrm{m}: \mathrm{B}_{\mathrm{m}} \subseteq \mathrm{X}\right\}=\bigcap_{\mathrm{seS}} \mathrm{X}_{\mathrm{s}}$. The deletion of a basin $\mathrm{X}$, with structuring template $\mathrm{B}$ is defined as the set of all points $m$ such that $B M$ intersects the $X$ as shown in $X \oplus B=\left\{m: B_{m} \cap X \neq \varphi\right\}=\bigcup_{s \in B} X_{s}$. A cascade of erosiondilation is called opening. The opening by a disk B, is the combination of erosion followed by dilation by $\mathrm{B}$ is shown in $\mathrm{X}$ o $\mathrm{B}=(\mathrm{X} \ominus \mathrm{B}) \oplus \mathrm{B}$. Closing of $\mathrm{X}$ by a template $\mathrm{B}$ is dilation by $\mathrm{B}$ followed by erosion by $\mathrm{B}$. Mathematically it is shown as $X \bullet B=(X \oplus B) \ominus B$. These latter two transformations, which are based on 
the former two transformations and are idempotent transformations. They are referred to as nonlinear transformations. The non-linearity is due to noninvertible property that these two-idempotent transformations possess. However, these two latter nonlinear transformations can be implemented iteratively by following multi-scale approaches, in which the size of the SE will be incremented from iteration to iteration. In the multi-scale approach, the size of the $B$ will be increased from iteration to iteration.

$$
n B=B \oplus B \oplus B \oplus \ldots \oplus B
$$

where, $\mathrm{n}$ is the discrete size parameter. Applying these basic morphological transformations, the various computations can be done with shapes. These transformations are systematically used in our study with an aim to characterize shapes by mean of pattern spectrum procedure.

\section{Skeleton Computation Via Morphological} Operations: The four fundamental transformations explained in earlier sub-section are used first to decompose a binary shape, akin to geophysical shape, and a skeleton (unique pattern ex: channel network), to reconstruct the shape of the skeleton. Subsequently the application of these transformations is shown in the pattern spectrum analysis as well as in the reconstruction accuracy analysis. A connectivity preserving way of erosion is termed as skeletonization. The skeleton is a one-picture element (pixel) thick object. That summarizes the overall shape, size, orientation, and association, of a geometric structure from which inferences can be drawn. Skeletons are of special interest because they reflect the structure of the original objects in their end pixels and vertices. Mathematical morphologists developed the concept of skeletonization $^{[4,5]}$. Morphologically the skeleton extraction phase described by ${ }^{[6]}$ can be achieved by connecting the basic morphological transformation ${ }^{[5,6]}$ as shown in equation $(2,3)$

$$
\begin{aligned}
& \mathrm{Sk}(\mathrm{X})=(\mathrm{X} \Theta \mathrm{nB}) \backslash\{[(\mathrm{x} \Theta \mathrm{nB}) \Theta \mathrm{B}] \oplus \mathrm{B}\} \\
& \mathrm{n}=0,1,2, \ldots, \mathrm{N} \\
& \mathrm{Sk}(\mathrm{X})=\bigcup_{\mathrm{n}=0}^{\mathrm{N}} \mathrm{Sk}_{\mathrm{n}}(\mathrm{X})
\end{aligned}
$$

Where:

$\mathrm{Sk}_{\mathrm{n}}(\mathrm{X})$ denotes the $\mathrm{nth}$ skeletal subset of shape (X). In the above expression, subtracting from the eroded versions of $\mathrm{X}$ their opening by $\mathrm{B}$ retains only the angular points. The union of all such possible points produces morphological skeletal network (SK). Using this skeletal network that could be decomposed from a given shape, original shape can be precisely reconstructed by means of the same structuring element that is used in the process of skeleton computations. Morphological reconstruction of the shape is done by means of several predefined morphological rules by changing their characteristics. It involves two steps, first we have to derive the skeleton (networks) of the shape. The skeleton is precisely decomposed from its shape by the morphological rule that is designed precisely. In the next phase, these skeletal subsets, decomposed ${ }^{[7]}$ from the shape are dilated by an explicit number of iterations by way of trying to reconstruct the shape outline by means of certain predefined morphological rule. The image reconstruction can be built by an infinite sequence of dilation and intersection, until the result reaches stability. In order to verify the accuracy in the shape that is reconstructed, by considering the skeleton network decomposed from the original shape, with reference to the original shape, a method that can quantify and characterize the reconstruction accuracy is revisited in the section that follows.

Pattern Spectrum and Study of Reconstruction Accuracy: In morphology, a quantitative measurement of the size distribution of objects in an image is given by pattern spectrum. This size distribution in the form of the pattern spectrum may be used for object recognition. The pattern spectrum procedure has some invariant properties over Fourier spectrum. The pattern spectrum $^{[8,9,10]}$ of size $n$ by a structuring element is defined as the pixel-wise difference between the target image morphological opened from a homothetic set of structuring element of size $\mathrm{n}$, and that opened by structuring element of size $n+1$. A procedure to reconstruct the image from different levels of dilated shapes is investigated ${ }^{[8,11,12,13,14]}$. The dilated portions are identified with the use of mathematical morphological SE with different scale. This method (Fig. 2) decomposes an object into a number of simple components based on homothetics of a set of SE. Mathematical morphological transformations are employed to decompose a binary shape by means of various SE. These procedures have been used in an integrated manner to study certain aspects of binary image. These aspects include the derivation of morphological rules of the topological structure of a binary shape, and vice versa. To derive these rules several binary shapes are simulated and the procedure based on mathematical morphology have been systematically implemented to verify the accuracy of morphological rules and in the reconstructed shapes [Eq. 4]:

$$
\begin{aligned}
& \mathrm{X}=\mathrm{U}_{\mathrm{n}=1}^{\mathrm{N}}\left[\mathrm{S}_{\mathrm{n}}(\mathrm{X}) \oplus \mathrm{nB}\right] \\
& \text { where } \mathrm{S}_{\mathrm{n}}(\mathrm{x})=((\mathrm{X} \Theta \mathrm{nB}) \backslash(\mathrm{X} \Theta \mathrm{nB}) \circ \mathrm{B})
\end{aligned}
$$


Pattern spectrum $\mathrm{PS}_{\mathrm{x}}(\mathrm{r}, \mathrm{B})$ is given by (5), where $\mathrm{A}(\mathrm{X})$ means that the area of $\mathrm{X}$ and $\mathrm{r}$ is the scale.

$\mathrm{PS}_{\mathrm{x}}(\mathrm{r}, \mathrm{B})=\mathrm{A}((\mathrm{X} \circ \mathrm{B}) \backslash(\mathrm{X} \circ \mathrm{rB})) \quad(\square \mathrm{rf} 1)$

$\mathrm{A}(\mathrm{X} \circ \mathrm{rB})$ is a measure of the pattern content of $\mathrm{X}$ relative to the pattern $\mathrm{rB}$. By varying both $\mathrm{r}$ (scale) and the shape of B (structuring element) we obtain a shape-size spectrum of $\mathrm{X}$, which is the full pattern spectrum of $X$ relative to all the patterns that can fit inside $X$. The higher a spectrum at the maximum $r$, the more alike an area $\mathrm{X}$ is as a structuring element $\mathrm{B}$. Shapiness is a likeness between $\mathrm{X}$ and $\mathrm{B}$. B-shapiness $\mathrm{S}_{\mathrm{x}}$ (B) [Eq. 6] is as follows, where $r_{\max }$ means that maximum of scale $r$ :

$$
\begin{aligned}
& \mathrm{S}_{\mathrm{X}}(\mathrm{B})=\mathrm{PS}\left(\mathrm{r}_{\max -1}, \mathrm{~B}\right) / \mathrm{A}(\mathrm{x}) \\
& \mathrm{S}_{\mathrm{X}}(\mathrm{B})=\left(\mathrm{PS}_{\mathrm{X}}\left(\mathrm{r}_{\max }, \mathrm{B}\right)+\mathrm{PS}_{\mathrm{X}}\left(\mathrm{r}_{\max -1}, \mathrm{~B}\right)\right) / \mathrm{A}(\mathrm{X})
\end{aligned}
$$

If $\mathrm{X}$ is completely similar to $\mathrm{B}, \mathrm{S}_{\mathrm{X}}(\mathrm{B})$ equal to 1 . The second equation is used when the spectrum at $r_{\max -1}$ is not maximum.

\section{RESULTS AND DISCUSSION}

Various planar shapes that include both classical and irregular shapes have been investigated in discrete space with an aim to estimate the shape-size complexity measure. To estimate this measure characteristic, the shape forms the points it shapes complexity with respect to the rule that has been used in the process of investigation in discrete space. In this study, we studied different shapes such as triangle, square, rectangle, octagon, hexagon, circle, and irregular shapes for reconstruction and accuracy of the reconstruction. At the n-1 scale it gives the maximum area. For SE it takes different scale required for convergence the same image in pattern spectrum. Fractal shape is considered with different SE. It gives 80 to $90 \%$ accuracy of the reconstruction. Square shape with square SE gives $100 \%$ accuracy. But with different SE it gives 10 to $40 \%$ of accuracy. Similarly the rectangle shape with square SE gives $100 \%$ accuracy but with other SE it gives 10 to $30 \%$ of accuracy. The shapiness index (Table 1) is a parameter that is used as a measure to compare the reconstruction accuracy of the binary shapes. The range of this index is 0 to 1 . If the shapiness index is 1 , then the two images are in the same pattern with exact geometric similarity. If it is 0 , then the two images are geometrically and topologically dissimilar. Incorporating various steps does the binary image reconstruction. Packing efficiency is based on the set and the complement ratio after achieving the full-length packing. The higher the ratio, the higher is the packing efficiency. It is hypothesized, which can be confirmed in quantitative terms, that the Fractal can be best packed with the highest packing efficiency, if we consider the Fractal type of structuring element, which should be homothetic of the Fractal. It is due to the fact that the shapiness index of the fractal by means of the both square and rhombus are 0.9 , which is very close to unity. In contrast, the shapiness index of the same Fractal by means of the octagon is 0.8 which can considered as a rule that describes the lower packing efficiency compared to the other two structuring elements.

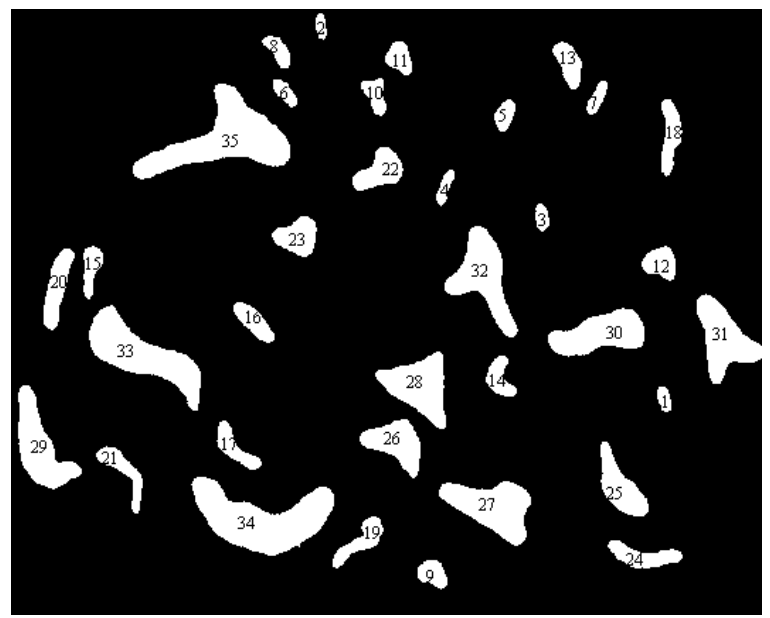

Fig. 1: Small Water Bodies, Traced from IRS 1D Remotely Sensed Data, Situated in the Flood Plain Region of Gosthani River (A.P) India
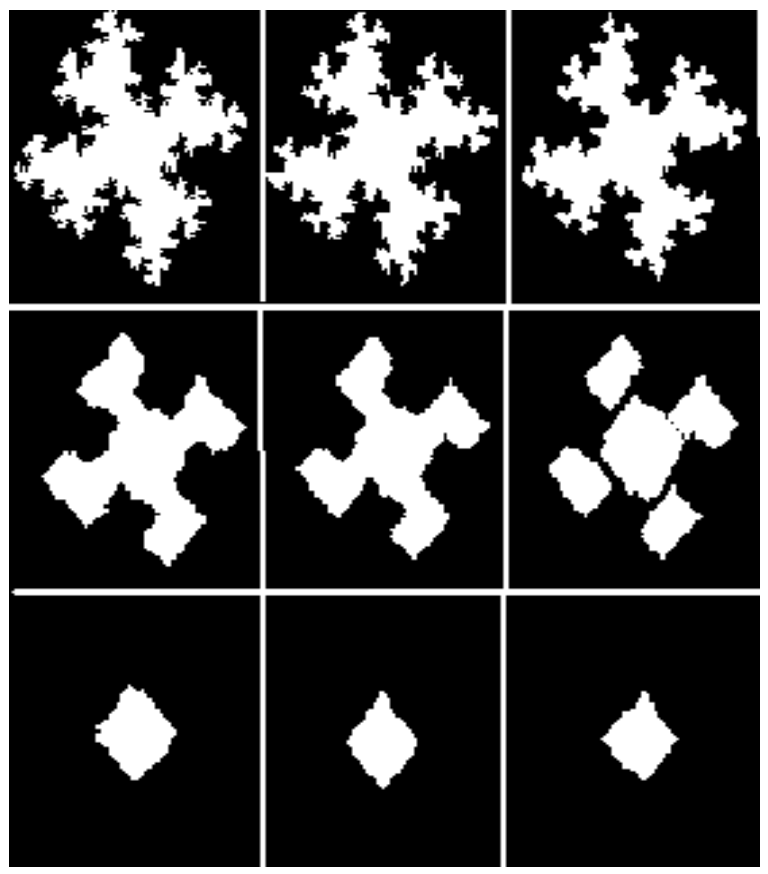

Fig. 2: Reconstructing Fractal Basin uses Rhombus as SE 
American J. Applied Sci., 2 (4): 843-846, 2005

Table 1: Shapiness Index of Various Shapes

\begin{tabular}{|c|c|c|c|c|c|c|c|c|}
\hline Shapes /SE & Square & Rectangle & Triangle & Octagon & Hexagon & Circle & Irregular Shape & Fractal \\
\hline SE(Square) & 1.0 & 1.0 & 0.5 & 0.6 & 0.6 & 0.4 & 0.6 & 0.9 \\
\hline SE(Octagon) & 0.1 & 0.1 & 0.4 & 1.0 & 0.4 & 0.9 & 0.8 & 0.8 \\
\hline SE(Rhombus) & 0.4 & 0.3 & 0.5 & 0.5 & 0.7 & 0.4 & 0.7 & 0.9 \\
\hline
\end{tabular}

Table 2: Shape-size Complexity of Lakes

\begin{tabular}{llllll}
\hline Name & Area & Shapiness & Name & Area & Shapiness \\
\hline Lake1 & 180 & 0.79 & Lake19 & 627 & 0.44 \\
Lake2 & 182 & 0.76 & Lake20 & 668 & 0.17 \\
Lake3 & 210 & 0.83 & Lake21 & 697 & 0.41 \\
Lake4 & 256 & 0.91 & Lake22 & 818 & 0.55 \\
Lake5 & 278 & 0.41 & Lake23 & 875 & 0.42 \\
Lake6 & 304 & 0.49 & Lake24 & 906 & 0.72 \\
Lake7 & 340 & 0.43 & Lake25 & 1051 & 0.40 \\
Lake8 & 362 & 0.50 & Lake26 & 1215 & 0.46 \\
Lake9 & 413 & 0.43 & Lake27 & 1779 & 0.36 \\
lake10 & 443 & 0.61 & Lake28 & 1792 & 0.42 \\
Lake11 & 454 & 0.53 & Lake29 & 1818 & 0.37 \\
Lake12 & 497 & 0.33 & Lake30 & 1822 & 0.27 \\
Lake13 & 512 & 0.39 & Lake31 & 2010 & 0.32 \\
Lake14 & 552 & 0.31 & Lake32 & 2022 & 0.42 \\
Lake15 & 555 & 0.46 & Lake33 & 2559 & 0.33 \\
Lake16 & 588 & 0.51 & Lake34 & 3326 & 0.27 \\
Lake17 & 604 & 0.57 & Lake35 & 4566 & 0.31 \\
Lake18 & 615 & 0.32 & & &
\end{tabular}

About 35 numbers of small water bodies (Fig. 1) situated randomly over a 10 sq. $\mathrm{km}$ landscape is considered to compute the shapiness indices with an aim to verify the general trend to derive a shape-size complexity relationships.

By employing the multiscale opening transformation, the implementation of which is shown on test water bodies, each water body's shapiness index is computed (Table 2). These shapiness indices range, for these water bodies, from 0.2 to 0.83 , indicates that smaller category water bodies possess higher shapiness indices indicating more regularity with morphology than that of larger water body categories. In this paper, ideas from the pattern spectrum procedure are employed to test the reconstruction accuracy in the basins. In addition to this, randomly situated surface water bodies of various sizes and shapes are also considered, and their shapiness indices are computed. A general trend is observed while characterizing the shape-size complexity of these surface water bodies.

\section{ACKNOWLEDGMENTS}

The author would like to acknowledge the excellent computational facilities provided by the Multimedia University, Malaysia and Assoc. Prof. B. S. Daya Sagar for his valuable guidance.

\section{REFERENCES}

1. Sagar B.S.D., M. Venu and D. Srinivas, 2000. Morphological operators to extract channel networks from digital elevation models. Intl. J. Remote Sensing, 21: 21-30.

2. Serra. J., 1984. Image Analysis and Mathematical Morphology. New York: Springer Verlag.

3. Goutsias, J., Henk and J.A.M. Heimans, 2000, Fundamental morphological mathematics. IOS Press, Fundamental Informatics 41: 1-33.

4. Lantuejoul, C., 1980. Skeletonization in quantitative metallography, in Issues of Digital image processing, R.M. Haralick and J.C. Simon, Eds. Groningen, The Netherlands: Sijthoff and Noordhoff.

5. Lantuejoul, C., 1982. Centre de Geostatistique et de Morphologie Mathematique. Int. Report, France.

6. Blum, H., 1973. Biological shape and visual sciences (Part I), J. Theor. Biol., 38: 205-287.

7. Pitas, J. and A.N. Venetsanopolos, 1990. Morphological shape decomposition. IEEE Trans. On Pattern Analysis and Machine Intelligence 12.

8. Maragos, P.A., 1989. Pattern spectrum and multiscale shape representation. IEEE Pattern Analysis and Machine Intelligence 11.

9. Omata, M. Hamamoto and T.S. Hangai, 2001. Lip Recognition Using Morphological Pattern Spectrum. Springer-Verlag AVBPA 2001, LNCS 2091, pp: 108-114.

10. Asano, A., M. Miyagawa and M. Fujio. Texture modeling by optimal gray scale structuring elements using morphological pattern spectrum, IEEE Trans. Pattern. Anal.

11. Reinhardt, J.M. and Q.E. Higgins, 1996. Efficient morphological shape representation. IEEE Trans. On Image Processing 5.

12. Sagar, B.S.D., M. Venu, G. Gandhi and D. Srinivas, 1998. Morphological description and interrelation between force and structure: A scope of geomorphic evolution process modeling. Int. J. Remote Sensing, 19: 1341-1358.

13. Tun-Wen Pai and J.H.L. Hansen, 1994. Boundaryconstrained morphological skeleton minimization and skeleton reconstruction. IEEE Trans. On Pattern Analysis and Machine Intelligence 16.

14. Maragos, P.A. and R.W. Schafer, 1986. Morphological skeleton representation and coding of binary images. IEEE Trans on Acoustics, Speech and Signal Processing, ASSP-34: 12281244. 